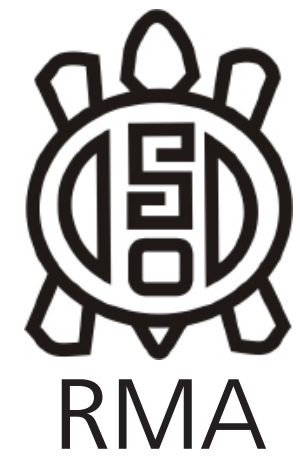

Arqueología

\title{
Estudio arqueobotánico de artefactos de molienda provenientes de la localidad arqueológica Punta Odriozola (golfo San Matías, Río Negro)
}

\author{
Archaeobotanical study of grinding stone tools from Punta Odriozola \\ archaeological locality (San Matias Gulf, Río Negro province)
}

María Laura Ciampagna*, Marcelo Cardillo** y Jimena Alberti***

* División Arqueología, FCNyM, Universidad Nacional de La Plata, Argentina. E-mail: mlciampagna@gmail.com

** CONICET, Instituto Multidisciplinario de Historia y Ciencias Humanas, Argentina. E-mail: marcelo.cardillo@gmail.com

*** CONICET, Instituto Multidisciplinario de Historia y Ciencias Humanas, Argentina. E-mail: jimealberti@gmail.com

\begin{abstract}
Resumen
En la localidad arqueológica Punta Odriozola (golfo San Matías) han sido relevados artefactos de molienda en distintos estados de preservación. Con el fin de caracterizar estos instrumentos y de determinar su posible funcionalidad, se relevaron sus caracteres métricos y las materias primas sobre las que fueron manufacturados, y se recolectaron los sedimentos preservados en las oquedades de dichos artefactos y en sus alrededores inmediatos. Los análisis arqueobotánicos arrojaron resultados positivos en uno de los casos, en el cual se identificaron granos de almidón de Prosopis sp. La presencia de este taxa en particular podría deberse a la molienda de las vainas de esta planta para uso comestible. La ampliación y refinación de los análisis permitirá determinar a futuro si estos artefactos pudieron haber sido utilizados para el procesamiento de otros recursos.
\end{abstract}

Palabras clave: Artefactos de molienda; Análisis arqueobotánicos; Golfo San Matías; Prosopis sp.

\begin{abstract}
In Punta Odriozola archaeological locality (San Matías Gulf) grinding stone tools showing different preservation status have been detected. In order to characterize these instruments and to determine their possible function, we have registered the lithic raw materials and the metric characters of the pieces, and we have collected the sediments preserved in their cavities and in their immediate surroundings. Archaeobotanical analysis yielded positive results in one of the cases, in which starch grains of Prosopis sp. were identified. The presence of these particular taxa could be due to the grinding of this plant pods for edible use. In the future, the enlargement of the analysis will allow us to determine if these artifacts were used to process other resources.
\end{abstract}

Keywords: Grinding stone tools; Archaeobotanical analysis; San Matías Gulf; Prosopis sp.

En el presente trabajo se exponen los primeros resultados del análisis de diez artefactos de molienda recuperados en la localidad arqueológica Punta Odriozola, costa oeste del golfo San Matías, Río Negro (Argentina) (Figura 1). La aplicación por primera vez de diversos enfoques y metodologías en el estudio de este tipo de instrumentos en el área permitió realizar una aproximación a su morfología y utilización en prácticas de procesamiento de recursos vegetales (Capparelli y Lema, 2010; Ciampagna, 2015).

En la Patagonia argentina se han realizado estudios de microrrestos vegetales recuperados en artefactos de molienda provenientes de los sitios arqueológicos de la provincia de Neuquén, Río Negro y Santa Cruz, con una cronología de hasta 3700 años AP de antigüedad (Lema et al., 2012). Estos estudios permitieron abrir la discusión en el área sobre el procesamiento de vegetales a través de la tecnología lítica. Estos resultados permitieron además, en relación al contexto de hallazgo, abordar categorías de uso alimenticio y medicinal de las plantas silvestres y domésticas (Ciampagna, 2012, 2015; Lema et al., 2012). En el presente trabajo se exponen los resultados de los 
análisis arqueobotánicos y tecno-morfológicos realizados en los instrumentos de molienda de Punta Odriozola con el objetivo de reconocer la utilización de los artefactos, así como identificar actividades de procesamiento de recursos vegetales silvestres.

\section{Descripción del área de estudio}

La costa oeste del golfo San Matías se extiende entre San Antonio Oeste y Puerto Lobos, en el límite con Chubut (ver Figura 1). La localidad arqueológica Punta Odriozola se emplaza sobre un pequeño campo de dunas que se habría formado sobre la antigua plataforma de abrasión del mar (Schellmann y Radtke, 2010). Esto habría favorecido la disponibilidad de agua en las depresiones entre médanos, hecho fundamental ya que en este tramo litoral no existen cursos de agua dulce permanentes o semipermanentes (Borella et al., 2015). En esta zona de la costa oeste se han relevado concheros asociados a concentraciones de material lítico. El Sector 3 de la localidad, que es el que resulta de interés en este trabajo debido a sus características ambientales ya mencionadas y a la distribución del registro (ver infra), se localiza a una distancia de aproximadamente 1.500 m desde la línea de costa actual, y presenta concheros muy erosionados sobre la superficie de deflación de una formación de dunas sobre una terraza. El registro recuperado incluye restos óseos, artefactos sobre valvas y líticos. Los materiales provenientes de la excavación de concheros que pudieron ser datados, arrojaron un fechado de 3.610 años ${ }^{14} \mathrm{C}$ AP (Borella et al., 2015), por lo que sería ésta la edad mínima de ocupación del área y, por lo tanto, de los artefactos de molienda allí encontrados. En este lugar, además, se registraron grabados (Carden y Borella, 2015, 2016) y una fuente primaria de toba silicificada (Alberti y Cardillo, 2015). A partir de análisis distribucionales se determinó

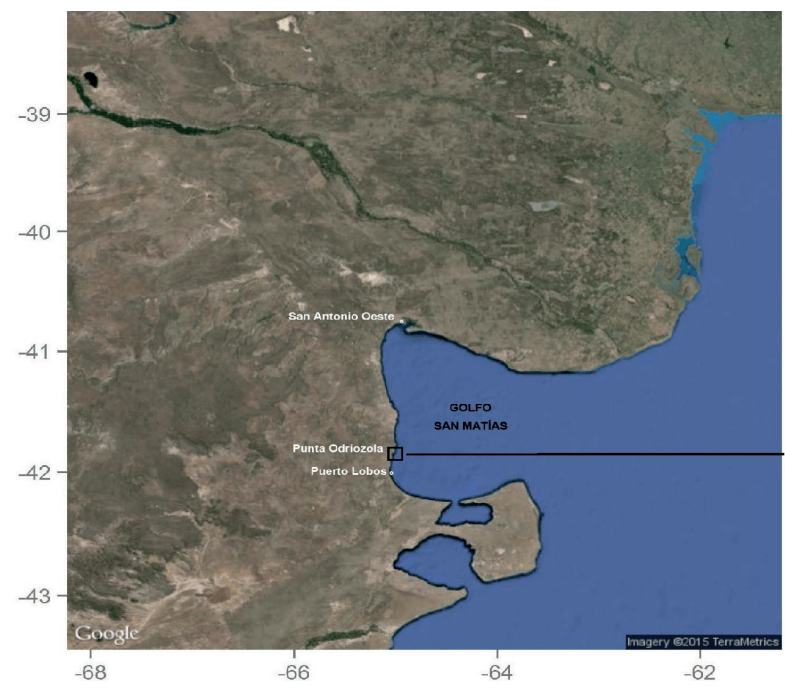

Figura 1. Ubicación de la localidad Punta Odriozola en la costa rionegrina. A la derecha se observa la distribución de los artefactos relevados en el Sector 3 de dicha localidad.

Figure 1. Punta Odriozola archaeological locality in the Río Negro province coast. In the right the artifact distribution from the Sector 3 is shown. 
En Punta Odriozola se registraron artefactos de molienda en su mayoría fragmentados (90\%) (Figura 2, Tabla 1). En algunos casos se observó perforación completa de la cara activa, sugiriendo un uso reiterado e intenso a lo largo del tiempo. La materia prima es una arenisca disponible localmente en los afloramientos rocosos situados directamente sobre la línea costera (Borella et al., 2015:243). La presencia de piezas agotadas y de superficies reactivadas sugeriría que estos artefactos, que demandan gran inversión de energía para su manufactura, fueron mantenidos lo cual implicaría una intención de reutilización a futuro (Cardillo et al. 2017). En relación con su distribución, los morteros se ubican sobre la terraza, cercanos a los concheros del Sector 3 y fueron recuperados todos en contextos de superficie. Este patrón podría sugerir espacios acotados de uso redundante a lo largo del tiempo, equipados con esta tecnología (Cardillo et al., 2017). Esto, sumado a la información cronológica general, podría indicar que los 3.000 años AP marcarían el inicio del empleo de esta tecnología.

\section{Materiales y métodos}

Se realizó el análisis de microvestigios de todos los artefactos de molienda recuperados en Punta Odriozola distribuidos sobre la terraza y cercanos al Sector 3 (Figura 1, Tabla1). Se siguió el protocolo de Ciampagna et al. (2011) y Ciampagna (2015) para la recuperación in situ de sedimento adherido en los artefactos. La extracción se realizó sobre oquedades de la cara activa del instrumento durante cinco minutos en cada uno de ellos (Babot, 2004; Lema et al., 2012; Ciampagna, 2015). En este caso, debido a las dimensiones y peso de los instrumentos, la recuperación del sedimento se debió llevar a cabo en el campo por lo que no se realizó una micro-estratigrafía de extracción y pipeteado con agua destilada (Ciampagna et al., 2011; Lema et al., 2012). Se guardó el sedimento circundante para obtener una muestra control con la cual comparar los residuos adheridos a los instrumentos. Esto último, sumado al protocolo descrito, es importante para trabajar con artefactos recuperados en contextos de superficie para así desestimar o corroborar casos de contaminación. La aridez y la salinidad del suelo, así como las escasas precipitaciones del ambiente donde estos artefactos fueron hallados, son variables que posibilitan la aplicación del estudio arqueobotánico en instrumentos de superficie. Además, su asociación a sitios concheros cuyo pH suele ser alcalino, medianamente alcalino e incluso neutro, constituye un ambiente favorable para la preservación de materia orgánica y, por lo tanto, de los granos de almidón y de los tejidos vegetales, entre otros (Babot, 2007; Hammond, 2015).

En el laboratorio se siguió el protocolo de Lema et al. (2012) y las soluciones fueron observadas en el microscopio óptico Leica DM//LM. Las muestras analizadas fueron de tipo "sucias" por lo que se registró la totalidad de microrrestos extraídos (Coil et al., 2003). El análisis se centró en los granos de almidón debido a que son diagnósticos taxonómicamente pero la totalidad de los elementos celulares fueron fotografiados y registrados (Lema et al., 2012). Este mismo procedimiento se realizó sobre una muestra del sedimento control. Los microrrestos recuperados fueron analizados cualitativamente.

Se relevaron los caracteres diagnósticos de los granos de almidón recuperados (Babot 2004, 2007, ICSN, 2011; Pagán Jiménez, 2015) y se compararon con una colección de referencia de 57 especies correspondientes a 29 familias de las provincias fitogeográficas del Monte y Patagonia (Ciampagna, 2015; Ciampagna et al., 2019) y con bibliografía pertinente (Giovanetti et al., 2008; Pérez y Erra, 2011; Ciampagna et al., 2011; Lema et al., 2012; Musaubach y Berón, 2012; Quiroz et al., 2012; Musaubach y Berón, 2016; Belmar, 2017; Saghessi y López, 2019).

\section{Resultados}

La muestra analizada en este trabajo está integrada por diez artefactos, en los cuales se relevaron las variables consignadas en la Tabla 1:

En la cara activa del $90 \%(n=9)$ de los instrumentos analizados se recuperaron hifas y esporas, y en el $60 \%(n=6)$ fibras y paquetes de fibras. Las primeras se observaron aisladas y en el interior de los elementos celulares vegetales. Por otra parte, en el $10 \%(n=1)$ de las muestras de sedimentos de control asociados a cada uno de los artefactos, se observaron paquetes de fibras,

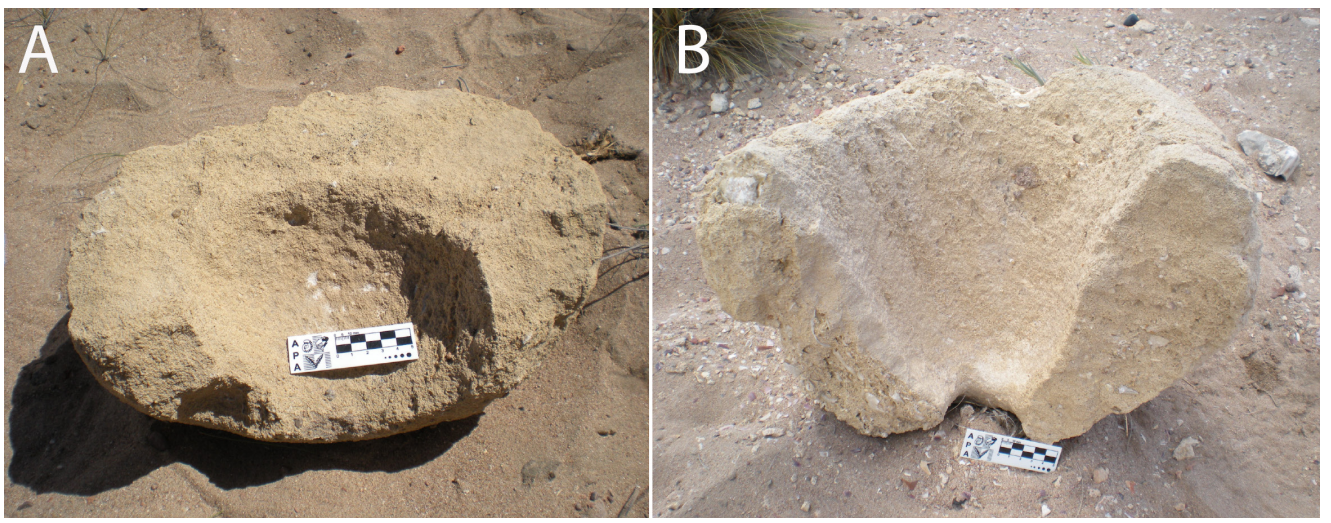

Figura 2. A) mortero con perforación por agotamiento. B) Mortero fragmentado con piqueteado de reavivamiento en su superficie interna.

Figure 2. A) Grinding stone tool perforated by use. B) Broken grinding stone tool with picket in its internal surface. 


\begin{tabular}{|c|c|c|c|c|c|c|c|c|}
\hline Sector & \# & $\begin{array}{c}\text { Materia } \\
\text { prima }\end{array}$ & Estado & $\begin{array}{l}\text { Largo } \\
(\mathrm{mm})\end{array}$ & $\begin{array}{c}\text { Ancho } \\
(\mathrm{mm})\end{array}$ & $\begin{array}{c}\text { Espesor } \\
(\mathrm{mm})\end{array}$ & $\begin{array}{l}\text { Peso } \\
\text { (kg) }\end{array}$ & Observaciones \\
\hline \multirow{5}{*}{ OD3 } & 1 & Arenisca & $\mathrm{Fg}$ & 300 & 250 & 50 & 10 & $\begin{array}{l}\text { Reactivación de } \\
\text { superficie }\end{array}$ \\
\hline & 2 & Arenisca & $\mathrm{Fg}$ & 180 & 120 & 90 & 4 & --- \\
\hline & 3 & Arenisca & $\mathrm{Fg}$ & 350 & 200 & 100 & 5 & $\begin{array}{l}\text { Reactivación de } \\
\text { superficie }\end{array}$ \\
\hline & 4 & Arenisca & Fg in situ & 300 & 200 & 130 & 25.5 & Agotado \\
\hline & 5 & Arenisca & $\mathrm{Fg}$ & 200 & 100 & 50 & 4 & ---- \\
\hline OD4 & 1 & Arenisca & Entero & 450 & 330 & 70 & 22 & $\begin{array}{l}\text { Piqueteado de } \\
\text { formatización }\end{array}$ \\
\hline OD5 & 1 & Arenisca & $\mathrm{Fg}$ & 400 & 300 & 110 & 28 & Agotado \\
\hline OD6 & 1 & Arenisca & Fg in situ & 570 & 330 & 70 & 18 & --- \\
\hline $\begin{array}{l}\text { PO } \\
\text { MORT }\end{array}$ & 1 & Arenisca & $\mathrm{Fg}$ & 300 & 300 & 70 & 18 & Agotado \\
\hline M1 & 1 & Arenisca & $\mathrm{Fg}$ & 300 & 250 & 50 & 9 & $\begin{array}{c}\text { Se encontraba con la } \\
\text { cara activa hacia } \\
\text { abajo }\end{array}$ \\
\hline
\end{tabular}

Tabla 1. Variables relevadas de la muestra analizada. $\mathrm{mm}$ : milímetros. Fg.: fragmentado. \#: número de pieza.

Table 1. Variables analyzed on the sample. mm: millimeters. Fg.: broken. \#: piece number.

como en el sedimento de control, son considerados elementos constitutivos del sedimento y por lo tanto una intrusión en el material lítico. En el instrumento 1 de OD3 se observó un elemento de vaso y esclereidas, que no se registraron en el sedimento de control pero que carecen

en el 20\% ( $n=2)$ fibras sueltas, y en el $40 \%(n=4)$ hifas y esporas. En la cara activa de uno de los instrumentos se recuperó un espodograma de células largas unidas, y en dos de los artefactos un fitolito elongado. En ambos casos, las evidencias no son diagnósticas para la identificación taxonómica. En el sedimento de control se recuperaron también un espodograma y un fitolito cono truncado de Poaceae. En el sedimento de control de OD5 se registró, además, un vaso silicificado y un fitolito alongado, ambos no diagnósticos para la identificación taxonómica (Figura 3G, H e I). Debido a que este tipo de microrrestos se recuperaron tanto en las caras activas de elementos diagnósticos taxonómicos (Figura 3B, C).

Sólo en el instrumento M1 se recuperaron granos de almidón simples, agrupados y solitarios de Prosopis sp, con un rango de tamaño de 6,25 a 18,75 $\mu \mathrm{m}$. Los de menor tamaño son esféricos de superficies redondeadas, hilio fusiforme con la cruz de polarización central y brazos lineales por lo que se asignan a la categoría A1a (sensu Giovannetti et al., 2008). También dentro de los granos regulares se recuperó uno ovoide, sin facetas de presión con la cruz de polarización central y brazos lineales correspondiente a la categoría A1b. Se identificaron
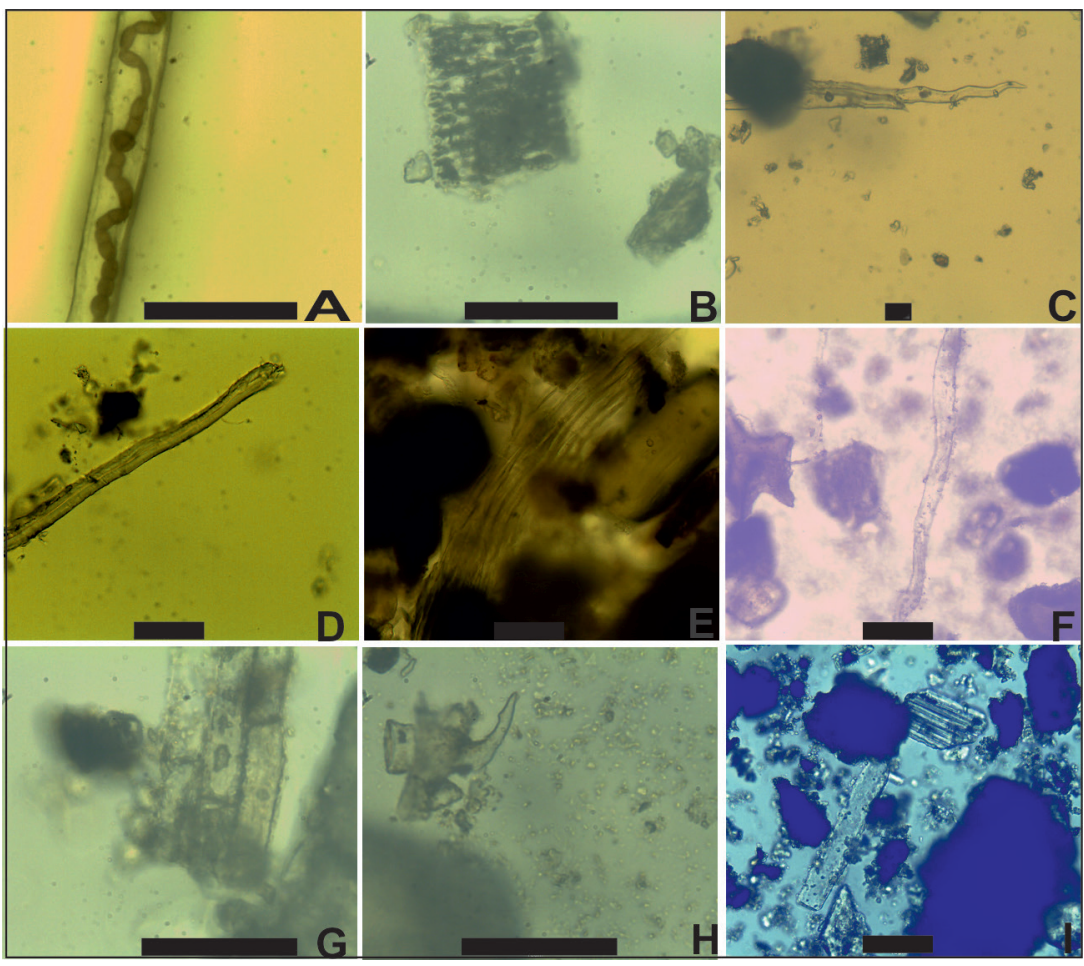

Figura 3. Figura 3. Microrrestos recuperados en las caras activas y en el sedimento control de los instrumentos. A-E: en las caras activas, hifas con esporas en su interior, elemento de vaso, esclereidas y fibras; $F$ : en el sedimento control, fibras e hifas; G: espodograma de células largas en cara activa y en sedimento control; $\mathrm{H}$ : cono truncado en el sedimento control; I: fitolito alongado en cara activa y sedimento control. La reglilla siempre representa $50 \mu \mathrm{m}$.

Figure 3: Microrests recovered in the active surfaces and in the sediment control of the tools. A-E on active surfaces: hyphae with spores inside, vessel element, sclereids and fibers; $F$ in the control sediment: fibers and hyphae. G: long cell spodogram on active surfaces and in control sediment; $\mathrm{H}$ : truncated cone in the control sediment; I: elongated phytolith in active surface and control sediment. The ruler always represents $50 \mu \mathrm{m}$. 
dos granos de superficies facetadas, con la cruz de polarización central y brazos lineales de categoría A2 y los restantes irregulares con más de una protuberancia o facetas irregulares con cruz de polarización excéntrica y brazos lineales. No se identificaron restos de tejido vegetal u otras células asociados a estos granos (Figura 4). En el instrumento POMORT no se hallaron microvestigios.

\section{Discusión y conclusiones}

En el conjunto de los instrumentos de molienda de Punta Odriozola se destaca la presencia de piezas realizadas sobre areniscas locales, que se encuentran agotadas o con sus superficies reactivadas. Estos rasgos sugerirían el mantenimiento de los artefactos y su reutilización, variables que, asociadas a la distribución espacial circunscripta y próxima a sitios concheros, darían cuenta de prácticas de equipamiento del espacio (Cardillo et al., 2017). En este sentido, los resultados obtenidos del estudio arqueobotánico son relevantes para la discusión sobre prácticas de procesamiento de plantas silvestres en sociedades cazadoras-recolectoras, así como sobre los procesos tafonómicos que pudieron intervenir en la preservación de los microrrestos.
El único artefacto en el que pudieron ser identificadas evidencias de algún tipo de actividad de molienda de restos vegetales fue el artefacto $M 1$, en el cual se recuperaron granos de almidón en agregado e individuales de Prosopis sp. sin que se hallara alguno en la muestra control. El hecho de que estos granos de almidón no se registraran en los otros instrumentos permitiría descartar la contaminación o intrusión de estos microrrestos. Es importante mencionar que los frutos de Prosopis tienen escasos granos de almidón, por lo que su recuperación en artefactos de molienda también se espera que sea escasa (Giovannetti et al., 2008; Lema et al., 2012). En los granos observados no hay evidencias de huellas de procesamiento, como podrían ser las fisuras o fracturas en su superficie, lo cual puede deberse a la manipulación de dichos granos a través del uso de molinos como es el caso de M1 (Babot, 2006; Lema et al., 2012).

En la Patagonia continental argentina la molienda de vainas secas y/o tostadas de algarrobo para la elaboración de harinas, así como mezcladas con agua o alcohol para la realización de bebidas se ha registrado a través de fuentes etnográficas y etnohistóricas (Ciampagna y Capparelli, 2012; Capparelli y Prates, 2015). En el sitio Angostura

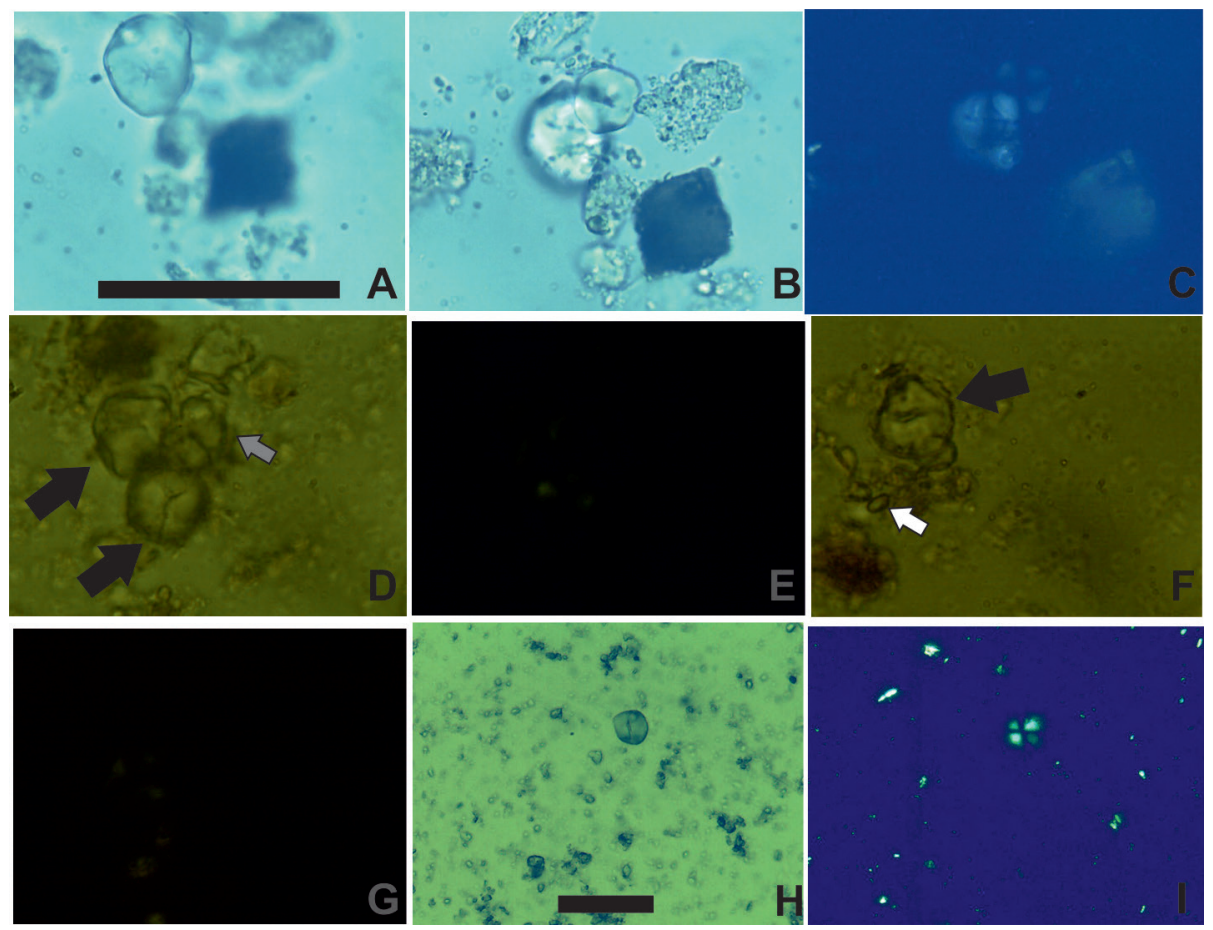

Figura 4. Granos de almidón recuperados en el molino M1. A: Tipo B2; B: tipo A2; C: polarización de los granos de almidón A y $B$; D: los granos pequeños esféricos señalados con una flecha gris son tipo A1a; E: polarización de los granos de almidón D; F: el grano más pequeño señalado con una flecha blanca es tipo A1b y los más grandes señalados con una flecha negra son tipo B2; G: polarización de los granos de almidón de la fotografía F; H: tipo A2 sin polarizar; I: polarización del grano de la foto I. La reglilla siempre representa $50 \mu \mathrm{m}$.

Figure 4. Starch grains recovered in the $M 1$ grinding tool. A: Type B2; B: type A2; C: polarization of starch grains $A$ and $B$; D: spherical small grains indicated with a gray arrow are type A1a; E: starch grains D polarization; $F$ : the smallest grain indicated with a white arrow is type A1b and the largest one indicated with a black arrow is type B2; G: polarization of the starch grains in photograph F; H: type A2 unpolarized; I: polarization of the grain in photo I. The ruler always represents $50 \mu \mathrm{m}$. 
1, en General Conesa y a $150 \mathrm{~km}$ de la costa atlántica, se recuperaron gran cantidad de vainas de Prosopis spp. que se habrían carbonizado accidentalmente al ser tostadas antes de consumir (Capparelli y Prates, 2015). El hallazgo de granos de almidón de Prosopis sp. en molinos de los sitios Aquihuecó y Michacheo (Neuquén), y en otros artefactos de la Costa Norte de Santa Cruz (junto a corpúsculos de resina en un molino de la colección de Museo del Hombre y asociados a pelos epidérmicos en un mortero de Punta Medanosa) son evidencia de que el procesamiento de algarrobo se encontraba espacialmente distribuido para el Holoceno tardío en Patagonia continental argentina (Ciampagna et. al., 2011; Ciampagna, 2012; Lema et. al., 2012). En la pampa occidental argentina también se han realizado estudios en los cuales han sido relevados microvestigios de Prosopis sp. entre otros recursos vegetales silvestres y domesticados (Musaubach y Berón, 2016).

En la costa del golfo San Matías no se han hallado aún vainas o carporrestos de este taxón, aunque sí se han identificado carbones provenientes de áreas de combustión de sitios concheros de Bajo de la Quinta desde 3.000 años cal. AP (Marcos y Ortega, 2014). Este registro permitiría sugerir que las poblaciones que habitaron el área conocían este recurso vegetal. La identificación de microrrestos y su procesamiento a través de la tecnología lítica permite ampliar las categorías de uso del algarrobo como combustible a su utilización al menos como alimento en el pasado. Estos resultados refuerzan la valoración de Moreno y Escobar (2001) y Gómez Otero (2007) sobre este recurso para la dieta de cazadores-recolectores de Patagonia.

Por otra parte, es relevante considerar qué factores pudieron haber intervenido en los instrumentos estudiados en los que no se hallaron granos de almidón. Es posible que procesos tafonómicos posdepositacionales, la proximidad a la costa y los vientos húmedos pudieran haber afectado la preservación, si bien como se mencionó anteriormente el $\mathrm{pH}$ de los suelos en sitios concheros, la aridez y las escasas precipitaciones serían condiciones propicias para el análisis arqueobotánico de instrumentos en superficie. En el caso del instrumento M1, la cara activa se encontraba hacia abajo, posición que pudo haberla preservado mejor que a los otros artefactos. Los granos de almidón y otros tejidos vegetales se conservan en suelos neutros o alcalinos, mientras que los fitolitos pueden degradarse parcialmente ante un precipitado de carbonato de calcio y diluirse en medios calcáreos con pH superior a 9 (Pearsall, 2000; Mussaubach et al. 2010). Esto puede estar indicando una preservación diferencial donde los granos de almidón se conservaron mejor en caso de que en los otros artefactos se hubieran procesado plantas productoras de fitolitos pero no de granos de almidón (i.e calcifitolito de Berberis, Belmar, 2017). Además, debe considerarse que la reactivación de los instrumentos de molienda pudo haber removido microvestigios si estos artefactos hubieran sido utilizados para el procesamiento de plantas o de otros recursos, tales como charqui y pigmentos, entre otros. Las hifas y esporas identificadas en el 50\% de las caras activas y de las muestras de sedimento de control, y los escasos fitolitos descriptos, así como las fibras y paquetes de fibras, serían producto de la intrusión de estos elementos al formar parte del sedimento donde se encontraban depositados los instrumentos. Las hifas y esporas halladas en el interior de elementos de vaso o fibras podrían corresponderse a endomicorrizas. Éstas son abundantes en suelos pobres como es el caso de las estepas, en ambientes dominados por plantas herbáceas y leñosas (Smith y Read, 1998, en Camargo Ricalde et al., 2012). Esto indicaría que los instrumentos estaban en un medio similar de depositación.

El análisis de los instrumentos de molienda del área de estudio y los resultados obtenidos permiten abordar en forma preliminar la utilización de recursos vegetales silvestres. Se deberá ampliar la muestra y establecer la recuperación en laboratorio de los microvestigios a partir de la incorporación de otras estrategias de recuperación de residuos a los fines de poder establecer hipótesis más amplias acerca del uso de los recursos por parte de las poblaciones cazadoras-recolectoras que habitaron el golfo San Matías durante el Holoceno medio y tardío.

\section{Agradecimientos}

A Jonathan Milinzoni y al equipo de investigación por su ayuda durante los trabajos de campo. A Aylen Capparelli y Laura López por sus sugerencias. Al CONICET y a la Agencia Nacional de Promoción Científica y Tecnológica por los subsidios que permitieron la concreción de estos trabajos. A los evaluadores anónimos cuyos comentarios ayudaron a enriquecer el manuscrito. Al equipo editorial de la revista por el trabajo realizado.

\section{Bibliografía}

Alberti, J. y Cardillo, M. (2015) Primary and secondary lithic raw material sources along the western coast of San Matías Gulf (Río Negro province, Argentina): a first approach to their spatial variability. Quaternary International 373: 63-71.

Babot, M. P. (2004) Tecnología y utilización de artefactos de molienda en el Noroeste Prehispánico. Facultad de Ciencias Naturales e I. M. L. Universidad Nacional de Tucumán, Tucumán. Tesis Doctoral no publicada.

Babot, M. P. (2007) Granos de almidón en contextos arqueológicos: posibilidades y perspectivas a partir de casos del Noroeste argentino. En Marconetto, B., Babot, P. y Oliszewski, N. (Eds.), Paleoetnobotánica del Cono Sur: estudios de casos y propuestas metodológicas, pp. 95-125. Centro Editorial de la Facultad de Filosofía 
y Humanidades, Universidad Nacional de Córdoba, Córdoba.

Belmar, C. (2017) Explotación de recursos vegetales entre grupos cazadores recolectores. Una aproximación desde el registro arqueobotánico Cueva Baño Nuevo 1 (Aisén, Chile). Tesis doctoral, Facultad de Ciecias Sociales, Universidad Nacional del Centro de la Provincia de Buenos Aires, Argentina.

Borella, F., Cardillo, M., Favier Dubois ,C. y Alberti, J. (2015) Nuevas investigaciones arqueológicas entre Punta Pórfido y Punta Odriozola: implicancias para el entendimiento de la dinámica de las ocupaciones humanas en la costa oeste del golfo San Matías (Río Negro). Relaciones de la Sociedad Argentina de Antropología 40(1): 233-252.

Camargo Ricalde, S., Montaño, M., De la Rosa-Mera, C. y Montaño Arias, S. (2012) Micorrizas: una gran unión debajo del suelo. Revista digital Universitaria 13(7): 3-19.

Capparelli, A. y Lema, V. (2010) Prácticas poscolecta/ post-aprovisionamiento de recursos vegetales: una perspectiva paleoetnobotánica integradora aplicada a casos de Argentina. Actas del XVII Congreso Nacional de Arqueología Argentina, Tomo III: 1171-1176.

Capparelli, A. y Prates, L.. (2015) Explotación de frutos de algarrobo (Prosopis spp.) por grupos cazadoresrecolectores del noreste de Patagonia. Chungara 47(4): 549-563.

Carden, N. y Borella, F. (2015) Symbols by the sea: the first recording of Atlantic coastal rock art in Patagonia (Punta Odriozola, Río Negro, Argentina). Rock Art Research 32: 146-162.

Carden, N. y Borella, F. (2016) Primeros datos sobre arte rupestre en el litoral atlántico de Patagonia continental. Los petroglifos de Punta Odriozola (Río Negro, Argentina). En Arqueología de la Patagonia: de mar a mar, pp. 421429. Ñire Negro, Santiago de Chile.

Cardillo, M., Alberti, J. y Carranza, E.. (2017) Tecnología, uso de materias primas y redundancia ocupacional: la localidad Punta Odriozola, costa de la provincia de Río Negro, Argentina. Mundo de Antes 11: 71-98.

Ciampagna, M. L. (2012) Prácticas poscolecta, molienda, de grupos cazadores-recolectores de la costa norte de Santa Cruz, Holoceno tardío. Trabajo presentado en las IX Jornadas de Jóvenes Investigadores en Ciencias Antropológicas. Buenos Aires.

Ciampagna, M. L. (2015) Estudio de la interacción entre grupos cazadores recolectores de Patagonia y las plantas silvestres: el caso de la Costa Norte de Santa Cruz durante el Holoceno medio y tardío. Facultad de Ciencias Naturales y Museo, Universidad Nacional de La Plata, La Plata. Tesis Doctoral no publicada.

Ciampagna, M. L. y Capparelli, A. (2012) Historia del uso de las plantas por parte de las poblaciones que habitaron la Patagonia continental argentina. Cazadoresrecolectores del cono Sur 6: 45-75.

Ciampagna, M. L., Molares, S., Ladio, A. y Capparelli, A. (2019) Use of wild Underground Storage Organs along the history of Patagonia: a contribution to macro and microarchaeobotanical visibility in hunter-gatherers Ms.

Ciampagna, M. L., Trola, V., Borrelli, L. y Capparelli, A. (2011) El uso de recursos vegetales por parte de grupos cazadores recolectores de la costa norte de Santa Cruz: análisis de instrumentos de molienda. Trabajo presentado en las VIII Jornadas de Arqueología de la Patagonia. Malargüe.

Coil, J., Korstanje, A., Archer, S. y Harstof, C. (2003) Laboratory goals and considerations for multiple microfossil extraction in archaeology. Journal of Archaeological Science 30: 991-1008.

Giovannetti, M., Lema, V., Bártoli, C. y Capparelli, A. (2008) Starch grains characterization of Prosopis chilensis (Mol.) Stuntz and P. flexuosa DC, and the analysis of archaeological remains in Andean South America. Journal of Archaeological Science 35: 2973-2985.

Gómez Otero, J. (2007) Dieta, uso del espacio y evolución en poblaciones cazadoras-recolectoras de la costa centroseptentrional de Patagonia durante el Holoceno medio y tardío. Facultad de Filosofía y Letras, Universidad de Buenos Aires, Buenos Aires. Tesis Doctoral no publicada.

Hammond, H. (2015) Sitios concheros en la costa norte de Santa Cruz: su estructura arqueológica y variabilidad espacial en cazadores recolectores Patagónicos. Facultad de Ciencias Naturales y Museo, Universidad Nacional de La Plata, Argentina. Tesis doctoral no publicada.

ICSN. (2011) The International Code for Starch Nomenclature. www.fossilfarm.org/ICSN/Code.html. Consultada el 30 de mayo de 2017.

Lema, V., Della Negra, C. y Bernal, V. (2012) Explotación de recursos vegetales silvestres y domesticados en Neuquén: implicancias del hallazgo de restos de maíz y algarrobo en artefactos de molienda del Holoceno tardío. Magallania 40(1): 229-249.

Marcos, M. A., Mancini, M. y Favier Dubois, C. (2012) Middle-Late Holocene environmental changes in Bajo de la Quinta, NE Patagonia, inferred by palynological records and their relation to human occupation. The Holocene 256(11): 1271-1281. 
Marcos, M. A. y Ortega, F. (2014) Paleoambientes y uso de los recursos leñosos por los grupos cazadores recolectores del noreste de Patagonia desde el Holoceno medio. Magallania 42(1): 147-163.

Moreno, J. E. y Escobar, F. (2001) Algarrobos, artefactos de molienda y cerámica en Patagonia centro sur: la distribución y el uso. Trabajo presentado en el XIV Congreso Nacional de Arqueología Argentina. Rosario.

Musaubach, M. G. y Berón, M. (2012) Cocinando en ollas en la Pampa Occidental. Datos desde la etnohistoria, el registro arqueológico y la arqueobotánica. En Las manos en la masa: arqueologías, antropologías e historias de la alimentación en Suramérica, pp. 605-626. ISES-CONICET, Córdoba.

Musaubach, M. G. y Berón, M. (2016) Food residues as indicators of processed plants in hunter-gatherers' pottery from La Pampa (Argentina). Vegetation history and archaeobotany 26(1): 111-123.

Mussaubach, M. G., Erra, G. y Osterrieth, M. (2010) Estudios arqueoetnobotánicos en la localidad Tapera Moreira (Departamento de Lihuel Calel, Provincia de La Pampa). Análisis fitolíticos en artefactos de molienda. En Berón, M., Luna, L., Bonomo, M., Montalvo, C., Aranda, C. y Carrera Aizpitarte ,M. (Eds.) Mamül Mapu: pasado y presente desde la arqueología pampeana Tomo I, pp. 77-86, Editorial Libros del Espinillo, Ayacucho, Argentina.
Orlando, M. (2009) Instrumentos de molienda y uso del espacio en la costa norte de la Provincia de Río Negro: una primera aproximación. En Salemme, M., Santiago, F., Álvarez, M., Piana, E., Vázquez, M. y Mansur, E. (Eds.), Arqueología de la Patagonia-Una mirada desde el último confín, pp. 1127-1140. Editorial Utopías, Ushuaia.

Pagán Jiménez, J. R. (2015) Almidones. Guía de material comparativo moderno del Ecuador para los estudios paleoetnobotánicos en el neotrópico. Aspha Ediciones, Buenos Aires.

Pearsall D. M. (2000) Paleoethnobotany: a handbook of procedures. Academic Press.

Quiroz, L., Belmar, C., Planella, T., Mera, R. y Munita, D. (2012) Estudios de microfósiles adheridos en pipas cerámicas del sitio Villa JMC-1, Labranza, Región de la Araucanía. Magallania 40(1):249-261

Saghessi, D. y López, L. (2019) Estudios de micorrestos vegetales en tártaro dental humano de sitios arqueológicos del Valle del Río Negro. En XX Congreso Nacional de Arqueología Argentina, Córdoba, Argentina.

Schellmann, G. y Radtke, U. (2010) Timing and magnitude of Holocene sea-level changes along the middle and south Patagonian Atlantic coast derived from beach ridge systems, litoral terraces and valley-mouth terraces. EarthScience Reviews 103: 1-30. 